\title{
1 Private Investoren zwischen Wohnungseigentums- und Einkommensteuerrecht
}

Der Wert einer Sache entspringt ihrem zukünftigen Nutzen. ${ }^{1}$ Aus finanzwirtschaftlicher Sicht dienen Investitionsobjekte als Mittel zum Zweck, ${ }^{2}$ um zukünftige Einnahmen zu generieren oder Auszahlungen zu vermeiden. ${ }^{3}$ In beiden Fällen geht es darum, nachhaltig eine befriedigende Verzinsung des bereitgestellten Kapitals zu erzielen. ${ }^{4}$ Bei Unternehmen besteht dieser Zukunftserfolg aus Gewinnausschüttungen, ${ }^{5}$ bei Eigentumswohnungen sind es Mietüberschüsse. ${ }^{6}$ Wird eine Wohnung selbst bezogen, besteht ihr Nutzen aus der ersparten Opportunitätsmiete für ein gleichwertiges Mietobjekt. ${ }^{7}$ Auch versprechen sich die Investoren oftmals langfristige Wertsteigerungen. ${ }^{8}$

Der Kauf einer Eigentumswohnung ist wirtschaftlich vorteilhaft, wenn ihr Wert größer als der gezahlte Preis ist. ${ }^{9}$ Jeder Investor hat eine individuelle Grenze, bei deren Überschreiten es nicht lohnt, weiter zu verhandeln. In der funktionalen Bewertungstheorie wird dieser kritische Preis Entscheidungswert oder Grenzpreis genannt. ${ }^{10}$ Aus Sicht des präsumtiven Käufers führt eine Transaktion oberhalb seines Entscheidungswerts - verglichen mit der Unterlassensalternative - zu wirtschaftlichen Nachteilen. ${ }^{11}$

1 Vgl. SCHMALENBACH (1917), S. 1, MÜNSTERMANN (1970), S. 151, HERING (2014), S. 27 f.

2 Vgl. WOLF (1886), S. 427, SIEBEN (1976), S. 497, GUTENBERG (1987), S. 1.

3 Vgl. AlbaCh (1962), S. 13, MATSCHKE/MATSCHKE (1993), S. 19, ADAM (2000), S. 4. Der Begriff stammt aus dem Lateinischen (investire) und bedeutet einkleiden, vgl. KLINGELHÖFER (2006), S. 8.

4 Dieses strategische Oberziel muß jedes Unternehmen zur Wahrung seines Fortbestands verfolgen, vgl. HERING (2017), S. 9, ähnlich BUSSE VON COLBE (1957), S. 24, MORAL (1919), S. 86. Dies gilt ebenfalls für jedes wirtschaftlich handelnde Individuum, denn „Unternehmer ist derjenige, der Investitions- und Finanzierungsentscheidungen trifft." SCHNEIDER (1992), S. 4.

5 Vgl. MOXTER (1983), S. 79, HeNSELMANN (1999), S. 79, TOLL (2011), S. 36. Zum Prinzip des Zukunftserfolgs vgl. BUSSE VON COLBE (1957).

6 Vgl. TOLL/WALOCHNIK (2013), S. 21, VON BRESSENSDORF (2016), S. 246, ROTTKE/EIBEL/KRAUTZ (2017), S. 18, PFLÜGL (2017e), Rn. 12 ff.

7 Vgl. RENNERT (2012), S. 18, LEIFELS/RAFFELHÜSCHEN (2014), S. 135. Selbstnutzer fokussieren sich im Investitionszeitpunkt jedoch häufig nicht auf die finanzielle Vorteilhaftigkeit, sondern auf ideelle Zielsetzungen wie bspw. Unabhängigkeit oder Selbstverwirklichung, vgl. SCHÜRT (2017), S. 75 f., MORING/MAIWALD/KEWITZ (2018), S. 79.

8 Vgl. SCHULTE/SOTELO/ALLENDORF/ROPETER-AHLERS/LANG (2016), S. 583, ROTTKE/EIBEL/KRAUTZ (2017), S. 27, FESSELMANN/KOBABE/SCHWENZFEIER/SIEGEMUND (2018), S. 52.

9 Vgl. MÜNSTERMANN (1970), S. 11 und S. 81, MEYERING (2007), S. 149 f., HERING (2014), S. 3.

10 Zum Entscheidungswert vgl. MATSCHKE (1975).

11 Vgl. JAENSCH (1966), S. 660, SIEBEN (1976), S. 496, LEUTHIER (1988), S. 9 f., BRÖSEL (2004), S. 517. In Bezug auf Immobilien SCHULTE/SOTELO/ALLENDORF/ROPETER-AHLERS/LANG (2016), S. 582.

(C) Der/die Autor(en) 2021

S. Walochnik, Bewertung von Eigentumswohnungen,

Finanzwirtschaft, Unternehmensbewertung \& Revisionswesen,

https://doi.org/10.1007/978-3-658-30840-7_1 
Es gilt also, (subjektiven) Wert und (potentiellen) Preis miteinander zu vergleichen. ${ }^{12}$ Es versteht sich von selbst, daß diese individuelle Preisgrenze nicht von einem allwissenden Markt vorbestimmt, sondern höchst persönlich und zudem subjektiv und situationsabhängig ist. ${ }^{13}$ Der präsumtive Käufer muß zukünftige Geldbewegungen prognostizieren und in einen heutigen Grenzpreis umformen. ${ }^{14}$ Dieser Vorgang heißt Bewertung: ${ }^{15}$ Es werden Modelle herangezogen und Berechnungen durchgeführt. Modelle sind vereinfachte Abbilder der Realität. ${ }^{16}$ Eine zu detailverliebte Abbildung wirkt sich einerseits lähmend auf das Modell aus. ${ }^{17}$ Andererseits setzen reale Entscheidungen eine realistische Modellumgebung voraus ${ }^{18}$ und erfordern die Würdigung situationsspezifischer Merkmale. Eine gute Modellierung zeichnet sich durch Komplexitätsreduktionen an der richtigen Stelle aus, während es relevante Aspekte scharf fokussiert. ${ }^{19}$

Mehr als Vorbereitung und Unterstützung bei realen, betriebswirtschaftlichen Entscheidungen kann kein Modell versprechen. ${ }^{20}$ Da sich viele Sachverhalte - wenn überhaupt - nur schwer in Zahlen ausdrücken lassen, ${ }^{21}$ wäre es unverantwortlich, sich bei der Entscheidung allein auf mathematisch berechnete Entscheidungswerte zu verlassen. ${ }^{22}$ Man muß sich der Vorteile, aber auch der Grenzen des angewandten Rechenwerks bewußt

12 Während der Wert die subjektive Preisgrenze beschreibt, ist der Preis ein (ex post) beobachtbares Verhandlungsergebnis, vgl. MÜNSTERMANN (1970), S. 151, ENGELEITER (1970), S. 15 f., MOXTER (1983), S. 5. Von gezahlten Preisen ausgehende Rückschlüsse sind nicht sinnvoll, weil sie wenig über die unterschiedlichen Wertvorstellungen sagen, deren Zusammenspiel oft der Beweggrund einer Transaktion ist, vgl. SCHMALENBACH (1917), S. 6, MELLEROWICZ (1926) S. 60, ENGELS (1962), S. 7.

13 Vgl. CONRAD (1912), S. 4, HERING/VINCENTI (2004), S. 343, HERING (2017), S. 310.

14 Vgl. HERING (2014), S. 4.

15 „Unter einer Bewertung wird die Zuordnung eines Wertes, zumeist in Form einer Geldgröße [...], zu einem Gegenstand - dem Bewertungsobjekt - durch das jeweilige Bewertungssubjekt verstanden. Als Bewertungssubjekt wird derjenige bezeichnet, aus dessen Sicht die Bewertung durchgeführt wird.“ MATSCHKE/BRÖSEL (2013), S. 3.

16 Vgl. SCHWEIM (1969), S. 28, MEADOWS/MEADOWS/RANDERS/BEHRENS (1974), S. 20 f., ROSENBERG (1975), S. 7, BITZ/EWERT/TERSTEGE (2018), S. 153 f., BAMBERG/COENENBERG/KRAPP (2019), S. 13.

17 Vgl. HERING (2002), S. 37, JAPES (2011), S. 26 f. und S. 43.

18 Vgl. HAX (1964), S. 430, TOLL/HERING (2017), S. 461.

19 Zu Komplexitätsreduktionen im Rahmen der Unternehmensbewertung vgl. BALLWIESER (1990).

20 Vgl. MATSCHKE (1975), S. 24 f., HAX (1985), S. 9, HERING/VINCENTI (2004), S. 343, HERING (2017), S. $399 \mathrm{f}$.

21 Vgl. SCHMALENBACH (1947), S. 22, MELLEROWICZ (1952), S. 13, HEIDER (1969), S. 81 ff.

22 Zu Kritik an blauäugigem Modellvertrauen vgl. HERING/OLBRICH/ROLLBERG (2010), S. 33, HAESELER/HÖRMANN (2010), S. 25 f. Kompetenz beginnt, ,wo die vereinfachten Modelle enden - bei ihren Anwendungsprämissen sowie den Grenzen ihrer Anwendbarkeit.“ HAESELER/HÖRMANN (2010), S. 25 f., ähnlich HERING/TOLL/SCHNEIDER (2012), S. 259, BITZ/EWERT/TERSTEGE (2018), S. 5. 
sein. ${ }^{23}$ Der Entscheider muß stets seinen gesunden Menschenverstand ${ }^{24}$ einsetzen, um auch andere, modellmäßig gar nicht erfaßte Faktoren zu berücksichtigen.

Bei der Bewertung sind viele spezielle Charakteristika zu berücksichtigen, die Immobilien mit sich bringen: ${ }^{25}$ Vor allem der mit der Standortgebundenheit einhergehende ausgeprägte Unikatscharakter schränkt die Vergleichbarkeit deutlich ein - und damit die Brauchbarkeit von Durchschnittswerten hinsichtlich Miete, Instandhaltungs- und Betriebskosten. Zahlreiche höchst individuelle Merkmale sind schwer meßbar, üben jedoch starken Einfluß auf den Zukunftserfolg aus: ${ }^{26}$ Die Zahlungsbereitschaft von Mietinteressenten wird bei jeder Neuvermietung von Eigenschaften wie Lage, Renovierungszustand oder Lichtverhältnissen beeinflußt. Man denke an eine gute Verkehrsanbindung, fußläufig erreichbare Geschäfte oder den günstig geschnittenen Grundriß.

Noch speziellere Bewertungsobjekte sind Eigentumswohnungen - sie bringen eine ganze Reihe an Besonderheiten mit sich. Ein vermietender Wohnungseigentümer kann aufgrund der Vorschriften des Wohnungseigentumsgesetzes nicht handeln, wie es ihm beliebt. Er befindet sich durch die mietvertragliche Bindung einerseits und die Stellung als Gesellschafter andererseits in zwei divergierenden Rechtssphären. ${ }^{27}$ Er ist beidseitig an völlig andere Rechte und Pflichten gebunden, die stellenweise kollidieren. ${ }^{28}$ Bspw. bestehen unterschiedliche Eigentumsverhältnisse an verschiedenen Gebäudeteilen. ${ }^{29} \mathrm{Im}$ Gegensatz zum ausdrücklich zugeordneten Sondereigentum an seiner Wohneinheit besitzt jeder Eigentümer nur einen ideellen Anteil am Gemeinschaftseigentum in Form

23 Manchmal kann man Abweichungen der Wirklichkeit von ihrer mathematischen Abbildung vernachlässigen, manchmal muß man gut hinsehen, vgl. DANTZIG (1966), S. 8, HAX (1985), S. 93.

24 „Denn ,das ist BWL“!“, HERING/ROLLBERG (2011), S. 58.

25 Unter Immobilien versteht man i.d.R. Grundstücke mit den darauf befindlichen Gebäuden, vgl. PFNÜR (2011), S. 5, GROMER (2012), S. 11, LINDAUER (2016), S. 1. Der Begriff stammt aus dem Lateinischen (immobilia bona) und bedeutet unbewegliches Gut, vgl. ebd. Zu den Merkmalen des Wirtschaftsguts Immobilie vgl. Unterabschnitt 2.2.3.1. Selbst bei zwei übereinander liegenden Wohnungen unterscheiden sich Licht- und Schallverhältnisse deutlich. Beispielsweise ist der städtische Lärm in höheren Etagen kaum noch zu vernehmen, oft ist dort von einer besseren Aussicht auszugehen. Zudem ist die Bewertungssituation von besonders vielen Unvollkommenheiten geprägt, vgl. ROPETER (1998), S. 10, GLEIßNER/JUST/KAMARAS (2017), S. 24.

$26 \mathrm{Vgl}$.HAAS (2010), S. 46, FELDMANN/GERSTNER/HOFMANN/ISENHÄFER/SEGERER/VÄTH(2016), S. 400, SCHARMANSKI/ WIENCKE (2017), S. 737 f., ERTLE-STRAUB (2019), S. $371 \mathrm{f}$.

27 Einen Überblick über dieses Problemfeld geben STÜRZER/KOCH/HOPFENSPERGER/STERNS/STERNSKOLBECK/ZIEGELMAYER (2016), S. 295 ff., SUILMANN (2017a), LEHMANN-RICHTER(2019), S. 105 ff., SUILMANN (2019).

28 Dieses Spannungsfeld läßt sich auch durch Gestaltung des Mietvertrags nur unvollständig beseitigen, vgl. SUILMANN (2017a), Rn. 25.

29 Auch zum Folgenden vgl. RENNERT (2012), S. 75, MÜLLER (2015a), Rn. 1, MUSIELACK (2017), S. 50. Sondereigentum kann durch Wohn- oder Gewerbeeinheiten sowie Garagen verkörpert werden. Für eine differenzierte Betrachtung sei auf Gliederungspunkt 2.2.3.2.1 verwiesen. 
seines prozentualen Miteigentumsanteils (MEA). ${ }^{30}$ Gleichwohl können Schäden am Gemeinschaftseigentum die Nutzbarkeit des Sondereigentums beeinträchtigen. Selbst wenn der Vermieter mietrechtlich zur Mangelbeseitigung verpflichtet ist, kann er die notwendigen Maßnahmen wohnungseigentumsrechtlich ggf. nicht kurzfristig durchsetzen. ${ }^{31}$ Der Tausch undichter Fenster oder die Reparatur einer maroden Abflußleitung kann als Sachbeschädigung strafbar sein, ${ }^{32}$ weil diese Gewerke - ebenso wie Dach, Heizung oder Wohnungstüren - zum Gemeinschaftseigentum gehören. ${ }^{33}$

Zur Vornahme von Reparaturen ist allein die Wohnungseigentümergemeinschaft (WEG) berechtigt. Obwohl deren Verwalter im Außenverhältnis vertretungsberechtigt ist, benötigt er grundsätzlich den Beschluß einer Eigentümerversammlung, bevor er Reparaturaufträge erteilt. ${ }^{34}$ Wartezeit und Folgekosten variieren in Abhängigkeit von Erfahrung und Qualifikation des Verwalters sowie Kooperationsbereitschaft und -fähigkeit der Miteigentümer. Wenn der Verwalter Termine verschleppt oder die anderen Eigentümer eine Beschlußfassung aus Unwissenheit oder Geldnot blockieren, kann dies negative finanzielle Auswirkungen haben, weil dem Mieter ungeachtet dessen Rechte zur Mietminderung zustehen..$^{35}$ Ein auf Eigentumswohnungen zugeschnittenes Bewertungsmodell muß in der Lage sein, derartige Merkmale auch bei fehlender Meßbarkeit in die Prognose zukünftiger Zahlungsströme einfließen zu lassen.

In der vorliegenden Arbeit wird die Perspektive einer Privatperson eingenommen, die den Kauf einer Eigentumswohnung in Erwägung zieht. Mit einem Anteil von rund 80 Prozent besitzen natürliche Personen den Großteil der deutschen Wohnimmobilien. ${ }^{36}$ Neben etwa 16 Mio. Selbstnutzern gibt es knapp 3,9 Mio. private Kleinvermieter. ${ }^{37}$ Diese besitzen im Schnitt 3,5 Wohnungen, erzielen durchschnittlich Mietüberschüsse von rund 750 EUR p.a. - und stammen aus allen gesellschaftlichen Schichten. ${ }^{38}$

30 Vgl. § 1 Abs. 2 WoEigG, vgl. dazu auch SCHNEIDER (2017), Rn. 1 ff., SCHULZE/STEIN/TIETGEN/ MÖLLER (2017), S. 119.

31 Vgl. dazu Gliederungspunkt 2.2.3.2.2.

32 Vgl. § 303 Abs. 2 StGB.

33 Zur Abgrenzung zwischen Sonder- und Gemeinschaftseigentum siehe Gliederungspunkt 2.2.3.2.1.

34 Vgl. BUB (2009), S. 245, SUILMANN (2018b), Rn. 38, GREINER (2019), Rn. 23 ff.

35 Vgl. MERLE (2018a), Rn. 131 f., SUILMANN (2019), S. 115.

36 Auch zum Folgenden vgl. STATISTISCHE ÄMTER DES BUNDES UND DER LÄNDER (2015), S. 92 f., INSTITUT DER DEUTSCHEN WIRTSCHAFT KÖLN (2017), S. 4 ff.

$37 \mathrm{Vgl}$. JUST/VOIGTLÄNDER (2017), S. 8 f.

38 Vgl. VOIGTLÄNDER (2014), S. 61, STATISTISCHES BUNDESAMT (2018b), S. 29. In Unterabschnitt 3.2.2.1 wird noch einmal auf diese statistischen Daten eingegangen. 
Viele dieser privaten Immobilieninvestoren beabsichtigen die Erzielung langfristig stabiler Mieterträge. ${ }^{39} \mathrm{Oft}$ wird im Rahmen der Altersvorsorge auch der Wunsch nach zusätzlichen Einkünften im Rentenalter oder einem späteren Selbstbezug genannt. ${ }^{40}$ Viele Anleger sehen in Immobilien einen Vermögensakkumulator und -speicher mit langfristiger Wertstabilität. ${ }^{41}$

Häufig werden auch steuerliche Vorteile genannt. ${ }^{42}$ Zwar wird die Zielsetzung der Steuerersparnis oft überstrapaziert oder absolut gesetzt, ${ }^{43}$ dennoch spielt die Berücksichtigung von Steuern bei der Bewertung eine wesentliche Rolle: ${ }^{44}$ Für den Investor zählt derjenige finanzielle Vorteil, der bei ihm ankommt und konsumiert werden kann, nämlich sämtliche Ein- und Auszahlungen, einschließlich Steuerlast. ${ }^{45}$ Überschüsse aus Vermietung und Verpachtung unterliegen nach $\S 21$ EStG der Einkommensteuer. ${ }^{46}$ Der für eine natürliche Person geltende Steuersatz steigt mit der Summe ihrer Einkünfte. Zur Berechnung dient die in $\S 32 \mathrm{a}$ EStG definierte Tariffunktion. Sie unterscheidet fünf Einkommensbereiche, in denen jeweils eine andere Rechenvorschrift anzuwenden ist. In zwei Abschnitten ist die Funktionsvorschrift nicht linear und führt zu einem Steuersatz, der mit zunehmendem Gesamteinkommen steigt, bis er bei einem Einkommen von 55.961 EUR wieder einen linearen Steuersatz von 42 Prozent erreicht und ab 265.327 EUR den Spitzensteuersatz von 45 Prozent annimmt. ${ }^{47}$

Angesichts ihrer relativen Höhe gehört die Einkommensteuer für private Vermieter zu den wichtigsten Immobiliensteuern. In der Bewertungsliteratur geht man jedoch - wenn überhaupt - von linearen Steuersätzen aus. ${ }^{48}$ Aus dem Blickwinkel von Kapitalgesellschaften trifft diese Vereinfachung durchaus zu. Den Besonderheiten von Eigentumswohnungen hingegen wird die Unterstellung eines konstanten Steuersatzes nicht

39 Vgl. TOLL/WALOCHNIK (2013), S. 21, RENNERT (2012), S. 4, WESTERHEIDE (2017), S. 494.

40 Vgl. LEIFELS/RAFFELHÜSCHEN (2014), S. 135, MÜTHLEIN/HOFFMANN (2017), S. 4.

41 Vgl. MARKMANN/MARKMANN/ROTTKE (2017), S. 636, ROTTKE (2017d), S. 840 f.

42 Vgl. ebd., MÜTHLEIN/HOFFMANN (2017), S. 4, LINDMAYER/DIETZ (2018), S. $323 \mathrm{ff}$.

43 Zur Kritik an der Überbetonung steuerlicher Aspekte, vor allem gegenüber Privatanlegern, vgl. BRÜBACH (2005), S. 74. Man kann ,niemals 100\% oder gar mehr“ der Verluste über Steuererstattungen kompensieren, warnt RENNERT (2012), S. 87. Aufgrund behaupteter Steuervorteile oder -vergünstigungen verringern viele Steuerpflichtige ihre Entscheidungsrationalität, kritisiert SCHNEIDER (1992), S. 321 f. Zur Verklärung der Zielsetzung Steuerersparnis vgl. ausführlich 4.5.3.

44 Steuern werden in $\S 3$ Abs. 1 AO definiert als Geldleistungen, die ohne Gegenleistung zu erbringen sind und zur Einnahmenerzielung des öffentlich-rechtlichen Gemeinwesens allen auferlegt werden.

45 Vgl. WAMELING (2004), S. 119 ff. und 168 ff., JAPES (2011), S. 33 ff., KRAFT/KRAFT (2018), S. 4 ff.

$46 \mathrm{Vgl}$. Abschnitt 3.1.1. Die Einkommensteuer ist eine Ertragsteuer, d.h., sie orientiert sich am erzielten wirtschaftlichen Ergebnis, vgl. NICKENIG (2018), S. 16.

47 Vgl. § 32a Abs. 1 EStG.

48 Vgl. z.B. ROPETER (1998), S. 300, MENSCH (2002), S. 186 ff., OBERMEIER/GASPER (2008), S. 130 ff., SCHIERENBECK/WÖHLE (2011), S. 344, SCHREIBER (2017), S. 673 ff., BITZ/EWERT/TERSTEGE (2018), S. 158. 
gerecht. Zwar liegt das durchschnittliche Haushalts-Nettoeinkommen deutscher Vermieter bei ca. 39.780 EUR pro Jahr ${ }^{49}$ Doch findet man in den Wirtschaftsrechnungen des STATISTISCHEN BunDESAMTS auch Haushalte mit Mieteinnahmen, deren jährliche Nettoeinkommen zwischen 20.400 und 31.200 EUR und damit deutlich niedriger liegen. ${ }^{50}$ Zwar sind Rückschlüsse vom Netto- auf das zu versteuernde Einkommen schwierig. Jedoch liegt nahe, daß dieses bei vielen Wohnungseigentümern unterhalb von 55.961 EUR und damit durchaus in den nichtlinearen Tarifzonen liegen kann. ${ }^{51}$ Investitionstheoretische Bewertungsmodelle sind häufig linear und damit inkompatibel zum stellenweise nichtlinearen Steuertarif. ${ }^{52}$

Obwohl die Modellierung einer realistischen Steuerlast problematisch ist, greift die Berücksichtigung eines konstanten Steuersatzes aus Sicht natürlicher Personen ebenso zu kurz wie die Ausblendung steuerlicher Effekte. Insbesondere wegen des langen Planungshorizonts von Immobilieninvestitionen wäre das Ergebnis verzerrt und der Entscheidungswert weniger verläßlich.

Die vorliegende Arbeit verfolgt das Ziel, das investitionstheoretische Zustands-Grenzpreismodell (ZGPM) ${ }^{53}$ weiterzuentwickeln, um den Entscheidungswert einer Eigentumswohnung aus Sicht einer natürlichen Person zu ermitteln. Im Hinblick auf eine Modellgestaltung, welche der Bewertungssituation möglichst gut angepaßt ist, soll durch spezifische Modifikationen erstmals ein Ansatz gebildet werden, der in der Lage ist, die nichtlineare Steuertariffunktion in einem betriebswirtschaftlichen Bewertungsmodell zu berücksichtigen.

Ferner sollen auf Ebene der Eingangsdaten die vielen, teils schwer quantifizierbaren Merkmale von Eigentumswohnungen erfaßt werden, denn neben der Einbeziehung realitätsnaher Rahmenbedingungen steht und fällt der Entscheidungswert mit der Qualität des Datenmaterials. ${ }^{54}$ Auch ein theoretisches Modell ist unnütz, wenn es wesentliche Eigenheiten des Bewertungsobjekts unberücksichtigt läßt. Es sind Prognosen erforderlich, wie die vielen einzelfallabhängigen Faktoren auf den Zahlungsstrom wirken.

49 Vgl. INSTITUT DER DEUTSCHEN WIRTSCHAFT KÖLN (2017), S. 14, dort als Monatswert von 3.315 EUR.

50 Vgl. STATISTISCHES BUNDESAMT (2018b), S. 29, auch dort als Monatswerte von 1.700 bis 2.600 EUR.

51 Man denke auch an steuerlich zusammen veranlagte Eheleute, deren nichtlinearer Einkommensbereich sogar bis ca. 112.000 EUR reicht, vgl. dazu Unterkapitel 4.8.

$52 \mathrm{Zu}$ Problemen bei der nichtlinearen Modellierung vgl. ELLINGER/BEUERMANN/LEISTEN (2003), S. 224, SUHL/MELLOULI(2013), S. 96,

53 Zum ZGPM vgl. HERING (2000a).

54 Vgl. BRÖSEL (2002), S. 74, ähnlich SIEBEN/SIELAFF (1989), S. 52, BALLWIESER (1990), S. 40, SIEGEL (1994), S. 2, ADAM (1996), S. 194, MATSCHKE/BRÖSEL (2013), S. 166, BRAUER (2019b), S. 397 f. Jedoch wird die „Abgrenzung und Quantifizierung der relevanten Zukunftserfolge [...] im Rahmen der Unternehmensbewertungstheorie bislang vernachlässigt.“ BRÖSEL/DECHANT (2003), S. 135. 
Jede Prognose erfolgt unter Unsicherheit. ${ }^{55}$ Da sich die realen Rahmenbedingungen immerzu verändern, existiert ex ante keine optimale Lösung. Die Höhe des Zukunftserfolgs ist ex ante unbekannt und hängt von vielen Faktoren, Umwelteinflüssen und Wechselwirkungen ab. Zudem steigt der Grad der Unsicherheit mit zunehmender zeitlicher Entfernung. ${ }^{56}$ So wichtig es auch wäre, mehrwertige Daten in die Bewertung einzubeziehen und transparent als Bandbreite offenzulegen, ${ }^{57}$ würde es doch den Rahmen der vorliegenden Ausführungen sprengen. Vielmehr soll vereinfachend von sicheren Erwartungen der zukünftigen Zahlungsströme ausgegangen werden, um die Ausführungen zu vereinfachen und exemplarisch greifbar zu machen.

Die Berücksichtigung von Unsicherheit soll in der vorliegenden Arbeit bewußt ausgespart werden, weil sie von den vielen bewertungsrelevanten Besonderheiten von Eigentumswohnungen sowie den steuerlichen Rahmenbedingungen ablenken würde. Es erscheint wichtig, sorgfältig zusammengetragene Eingangsdaten auf dem Fundament eines soliden Modells zu bewerten, zumal unter Sicherheit optimale Ergebnisse unter Unsicherheit zumindest als Ausgangspunkt für Heuristiken brauchbar sind. ${ }^{58}$ Daher soll vereinfachend von einwertigen Zukunftserwartungen ausgegangen werden.

Im folgenden Gang der Untersuchung stellt Kapitel zwei die Komponenten vor, aus denen der Zahlungsstrom einer Eigentumswohnung besteht. Es sollen verschiedene Datenquellen aufgezeigt werden, die zu ihrer Prognose dienen können. Zusätzlich wird mit der Nutzwertanalyse eine leicht handhabbare Methode vorgestellt, um die scheinbar fehlende Quantifizierbarkeit mit einem einfachen und universell anwendbaren Werkzeug zu überwinden.

Darauf folgend wird das ZGPM als zielführendes investitionstheoretisches Totalmodell vorgestellt, wobei Kapitel zwei die Einkommensteuer noch ausblendet. Die Aufmerksamkeit des Lesers soll der investitionstheoretischen Modellierung und den rechtsformspezifischen Besonderheiten des Bewertungsobjekts Eigentumswohnung gelten. Dieses didaktisch vereinfachte Kapitel kommt der vereinfachten Sicht selbst nutzender Wohnungseigentümer am nächsten. Deren ersparte Auszahlungen einer Opportunitätsmiete sind grundsätzlich keine steuerpflichtigen (Vermietungs-) Einkünfte. ${ }^{59}$

\footnotetext{
55 Zum Folgenden vgl. KEUPER/PAPE (2008), S. 586 ff., HERING/SCHNEIDER/TOLL (2011), S. 405 f., HERING (2017), S. 11 ff., S. 20.

56 Vgl. ADAM (1996), S. 42, MANDL/RABEL (1997), S. 211, JAPES (2011), S. 49 f.

57 Vgl. HERING (2017), S. 273 ff., ZWIRNER/PETERSEN (2017a), S. 181.

58 Vgl. HERING (2017), S. 15.

59 Vgl. VAN SUNTUM/SCHULTEWOLTER (2014), S. 195.
} 
Die Betrachtung wird in Kapitel drei um Einkommensteuern erweitert. Weil die steuerrechtliche Bemessungsgrundlage bestimmte Teile des investitionstheoretischen Zahlungsstroms nicht oder nicht zeitgleich erfaßt, ${ }^{60}$ wird zunächst beleuchtet, wie die Bemessungsgrundlage im Rahmen einer Nebenrechnung um diese Abweichungen bereinigt werden kann, um darauf basierend die Steuerlast zu berechnen. Mit der linearen Approximation wird daraufhin ein einfacher Weg vorgestellt, die nichtlineare Steuertariffunktion dem linearen Modell zugänglich zu machen.

Schließlich erfolgt in Kapitel vier ein Zuschnitt des Modells auf verschiedene Spezialfälle aus dem Bereich des Wohnungseigentums bzw. des Steuerrechts. Es werden unterschiedliche Modifikationen vorgestellt.

Der erste Umbau dient der Erfassung von Abweichungen des Kostenverteilschlüssels zwischen mietvertraglicher Betriebskostenabrechnung und WEG-rechtlicher Jahresabrechnung. Anschließend erfolgt eine Würdigung der WEG-Instandhaltungsrücklage, die investitionstheoretisch sofort, steuerlich aber erst zeitversetzt zu erfassen ist.

Es folgt eine Betrachtung der Rückzahlungsmodalitäten bei annuitätischen Immobiliendarlehen, insbesondere deren Tilgungsbeschränkungen. Anschließend werden mit einem fiktiven Verbot der Verlustverrechnung negativer Vermietungseinkünfte sowie der Modellierung steuerlicher Verlustvorträge zwei Ausprägungen steuerlicher Verlustverrechnung betrachtet.

Es folgen eine modellmäßige Berücksichtigung des steuerlichen Ansatzwahlrechts größerer Erhaltungsaufwendungen und die Würdigung des Vermieter-Mieter-Dilemmas: Sinnvolle Modernisierungsmaßnahmen erhöhen den Wohnkomfort des Mieters, werden aber vom Vermieter gezahlt. Dieser darf im Gegenzug die Jahresmiete um acht Prozent der aufgewendeten Kosten erhöhen. Das Zustands-Grenzpreisvektormodell (ZGPVM) ${ }^{61}$ wird eingesetzt, um die Schmerzgrenze des Vermieters zu berechnen: Welche Mieterhöhung unterhalb der acht Prozent-Grenze müßte er mindestens fordern, um keinen wirtschaftlichen Nachteil zu erlangen?

Im vorletzten Unterkapitel wird der Einkommensteuer-Grundtarif durch den für Eheleute geltenden Zusammenveranlagungstarif ersetzt. Im letzten Unterkapitel wird eine Verallgemeinerung des Modells vorgenommen, um die Sichtweise von Kapitalgesellschaften mit zwei unterschiedlichen Bemessungsgrundlagen und deren Besteuerung anhand von konstanten Steuersätzen zu ermöglichen.

${ }^{60}$ Wichtig sind in diesem Kontext Abschreibungen, vgl. HAX (1985), S. 114 f., JAPES (2011), S. 35, BUSSE VON COLBE/WITTE (2018), S. 85.

${ }^{61}$ Zum ZGPVM vgl. TOLL (2011). 
Ein reales Beispiel, das dem Verfasser aus seiner freiberuflichen Tätigkeit bekannt ist, wird die Ausführungen flankieren. ${ }^{62}$ Kapitel vier schließt mit einer Übersicht der modelltechnischen Abwandlungen, die zur Abbildung der verschiedenen Spezialfälle vorgenommen wurden.

Hauptaufgabe der Investitionstheorie ist die Unterstützung betriebswirtschaftlicher Entscheidungen unter realistischen, unvollkommenen Bedingungen. ${ }^{63}$ Vor diesem Hintergrund gibt Kapitel fünf einen zusammenfassenden Überblick über die zahlreichen Unwägbarkeiten, die bei der Bewertung von Eigentumswohnungen auftreten können. Außerdem erfolgt eine abschließende Würdigung von Ergebnissen der verschiedenen Modellvarianten und deren Bezug zueinander.

Open Access Dieses Kapitel wird unter der Creative Commons Namensnennung 4.0 International Lizenz (http://creativecommons.org/licenses/by/4.0/deed.de) veröffentlicht, welche die Nutzung, Vervielfältigung, Bearbeitung, Verbreitung und Wiedergabe in jeglichem Medium und Format erlaubt, sofern Sie den/die ursprünglichen Autor(en) und die Quelle ordnungsgemäß nennen, einen Link zur Creative Commons Lizenz beifügen und angeben, ob Änderungen vorgenommen wurden.

Die in diesem Kapitel enthaltenen Bilder und sonstiges Drittmaterial unterliegen ebenfalls der genannten Creative Commons Lizenz, sofern sich aus der Abbildungslegende nichts anderes ergibt. Sofern das betreffende Material nicht unter der genannten Creative Commons Lizenz steht und die betreffende Handlung nicht nach gesetzlichen Vorschriften erlaubt ist, ist für die oben aufgeführten Weiterverwendungen des Materials die Einwilligung des jeweiligen Rechteinhabers einzuholen.

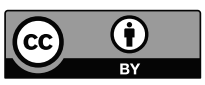

${ }^{62}$ Es handelt sich um die Bewertung einer Eigentumswohnung in Viersen im Jahr 2019. Nicht nur aus Datenschutzgründen, sondern auch zu Vereinfachungszwecken wurden viele Parameter modifiziert, schließlich soll die theoretische Modellierbarkeit im Vordergrund stehen.

${ }^{63}$ Vgl. HAX (1964), S. 430, HERING (2014), S. 304, MATSCHKE (2017a), S. 25. 